\title{
UTILIZAÇÃO DE ADSORVENTE ALTERNATIVO DE BAIXO CUSTO PARA REMOÇÃO DO CORANTE TARTRAZINA
}

\author{
E. L. FOLETTO* ${ }^{*}$, C. T. WEBER ${ }^{1}$, G. C. COLLAZZO ${ }^{2}$, M. A. MAZUTTI ${ }^{1}$, R. C. KUHN ${ }^{1}$; G. L. \\ DOTTO $^{1}$ \\ ${ }^{1}$ Departamento de Engenharia Química, Universidade Federal de Santa Maria \\ ${ }^{2}$ Centro de Ciências Exatas e Tecnologia, Universidade de Caxias do Sul \\ *E-mail para contato:efoletto@gmail.com
}

\begin{abstract}
RESUMO - Atualmente, buscam-se adsorventes alternativos para diminuir os custos do processo de adsorção, principalmente para tratamento de água residuárias. Neste trabalho utilizou-se semente de mamão cominuída (Carica papaya L.) para remoção do corante tartrazina de efluente sintético. Os efeitos do $\mathrm{pH}$, concentração inicial de corante e tempo de contato foram investigados. Os dados de equilíbrio foram analisados pelas isotermas de Langmuir, Freundlich e Temkin. A cinética foi avaliada de acordo com os modelos de pseudo-primeira ordem, pseudo-segunda ordem e Elovich. Verificou-se que o modelo de Langmuir foi o mais adequado para representar a operação, resultando em uma capacidade máxima de adsorção de $51 \mathrm{mg} \mathrm{g}^{-1}$. A cinética foi mais bem representada pelo modelo de pseudo-segunda ordem. Assim, os resultados demonstraram que as sementes de mamão são adsorventes alternativos de baixo custo com capacidade satisfatória para remover o corante tartrazina.
\end{abstract}

\section{INTRODUÇÃO}

Várias indústrias tais como, farmacêutica, têxtil e alimentícia, geram efluentes contendo corantes. Estes efluentes, quando descartados no meio ambiente, podem causar vários efeitos no ecossistema aquático e na vida humana (Foletto et al., 2013a). A operação de adsorção usando carvão ativado tem sido amplamente utilizado para tratar águas residuais contendo corantes (Foletto et al., 2012; Ioannou \& Simitzis, 2013; Zhang \& Ou, 2013; Linhares et al., 2013), no entanto, é relativamente caro devido ao custo de produção do carvão ativado. Portanto, materiais de baixo custo provenientes de resíduos industriais ou vegetais podem ser utilizados como adsorventes de corantes (Ramakrishna \& Viraraghavan, 1997, Weng et al., 2001; Yang et al., 2013) a fim de tornar a operação de adsorção mais acessível. Estudos recentes relatam que sementes de mamão possuem uma capacidade de adsorção altamente satisfatória para remoção de corantes. Estudos utilizando sementes de mamão como adsorvente de azul de metileno (Hameed, 2009; Paz et al., 2013), corantes da indústria coureira (Weber et al., 2013) e corantes têxteis (Foletto et al., 2013b) têm sido relatados recentemente. No entanto, a utilização de sementes de mamão para a adsorção de corantes farmacêuticos ainda é pouco relatada na literatura. $\mathrm{O}$ uso de sementes de mamão como adsorvente é muito interessante, uma vez que este é um resíduo agrícola de baixo custo. 
Neste trabalho, o corante tartrazina foi escolhido como um composto modelo para o estudo de adsorção. Tartrazina é um corante azóico com um grupo ácido sulfônico. É amplamente utilizado para tingir drogas e cosméticos (Gutpa et al., 2010). Assim, o objetivo deste trabalho foi avaliar o uso de sementes de mamão para a remoção do corante farmacêutico tartrazina a partir de solução aquosa sintética. Estudos de equilíbrio e cinéticos foram realizados com o objetivo de elucidar o processo de adsorção de corante sobre as sementes de mamão.

\section{METODOLOGIA}

\subsection{Adsorventes e adsorbatos}

As amostras de sementes de mamão foram preparadas e caracterizadas em um trabalho anterior (Foletto et al., 2013b). Os mamões foram obtidos em um comércio local. As sementes foram removidas do fruto e secas a $85^{\circ} \mathrm{C}$ em um forno durante $12 \mathrm{~h}$, seguida por trituração em moinho de facas. O material resultante foi peneirado, e uma porção com diâmetro de partícula entre 350 e 450 $\mu \mathrm{m}$ foi utilizada nos experimentos. A amostra apresentou uma baixa área superficial (BET), 0,23 $\mathrm{m}^{2}$ $\mathrm{g}^{-1}$, e diâmetro de poro médio de 332 A (Foletto et al., 2013b). Tartrazina (CAS 1934-21-0; formula química é $\mathrm{C}_{16} \mathrm{H}_{9} \mathrm{~N}_{4} \mathrm{Na}_{3} \mathrm{O}_{9} \mathrm{~S}_{2}$; peso molecular $=534.36 \mathrm{~g} \mathrm{~mol}^{-1}$ ), um corante amplamente utilizado na indústria farmacêutica, foi utilizado como composto modelo.

\subsection{Testes de adsorção}

Para os testes de adsorção, $0,05 \mathrm{~g}$ de adsorvente foram adicionados a $100 \mathrm{~mL}$ de solução aquosa com diferentes concentrações iniciais de corante $\left(20\right.$ a $\left.70 \mathrm{mg} \mathrm{L}^{-1}\right)$. Os ensaios de adsorção foram realizados em diferentes pHs $\left(2,5\right.$ a 8,5), os quais foram ajustados utilizando $\mathrm{H}_{2} \mathrm{SO}_{4}$ e $\mathrm{NaOH}$. A solução resultante foi agitada continuamente usando um agitador termostático (Tecnal, Brasil), até atingir o equilíbrio de adsorção, a uma temperatura constante $\left(25^{\circ} \mathrm{C}\right)$. Alíquotas da solução aquosa foram retiradas em vários intervalos de tempo, centrifugadas e filtradas antes da análise. As concentrações de corantes em solução aquosa foram determinadas por espectrofotometria (Spectro vision modelo T6-UV) em $\lambda_{\text {máx }} 426 \mathrm{~nm}$. A capacidade de adsorção do corante foi calculada como segue:

$$
q t=\left[\left(C_{o}-C_{t}\right) \cdot \mathrm{V}\right] / \mathrm{W}
$$

onde: $q t\left(\mathrm{mg} \mathrm{g}^{-1}\right)$ é a quantidade de corante adsorvido no tempo de contato $t(\mathrm{~min}), C o\left(\mathrm{mg} \mathrm{L}^{-1}\right)$ é a concentração inicial de corante, $C_{t}$ é a concentração de corante no tempo $t, W$ (g) é a massa de adsorvente na solução, e $V$ (L) é o volume da solução. Todos os ensaios de adsorção foram realizados em duplicata e só os valores médios foram reportados. O desvio máximo observado foi de cerca de $\pm 7 \%$.

\section{RESULTADOS E DISCUSSÃO}

\subsection{Efeito do pH sobre a adsorção}


$\mathrm{O}$ pH é um dos parâmetros mais relevantes na operação de adsorção, pois pode afetar as propriedades da superfície do adsorvente e a estrutura do adsorbato. O efeito do $\mathrm{pH}$ sobre a adsorção do corante foi estudado no intervalo entre 2,5 e 8,5 (Fig. 1). A partir da figura, observou-se que a adsorção foi elevada em meio ácido $(\mathrm{pH}$ 2,5). Um baixo $\mathrm{pH}$ leva a um aumento da concentração de íons $\mathrm{H}^{+}$no sistema e, a superfície do adsorvente adquire uma carga positiva por adsorver os íons $\mathrm{H}^{+}$. Como a superfície do adsorvente é possivelmente carregada positivamente a um pH baixo, uma forte atração eletrostática ocorre entre a superfície de adsorvente carregada positivamente e a molécula de corante aniônico $\left(\mathrm{SO}^{3-}\right)$. À medida que o $\mathrm{pH}$ do sistema aumenta, o número de sítios carregados negativamente aumenta, favorecendo a repulsão das moléculas de corante (Malik, 2004).

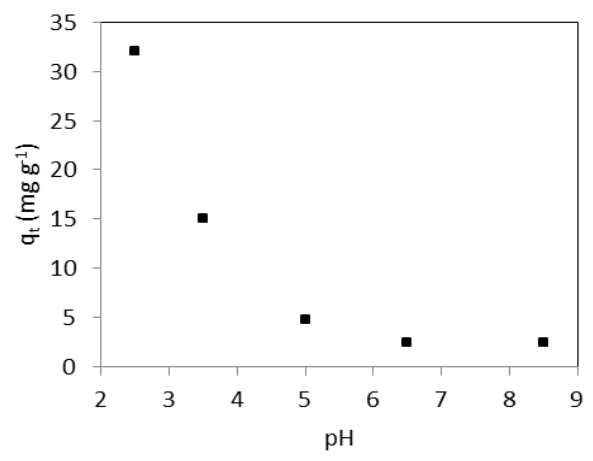

Figura 1 - Efeito do $\mathrm{pH}$ na adsorção de corante $\left(\mathrm{C}_{\mathrm{o}}=20 \mathrm{mg} \mathrm{L}^{-1}\right)$ sobre a semente de mamão a $25{ }^{\circ} \mathrm{C}$.

\subsection{Efeito da concentração inicial de corante e tempo de contato}

As influências do tempo de contato e da concentração na adsorção do corante são mostradas na Figura 2.

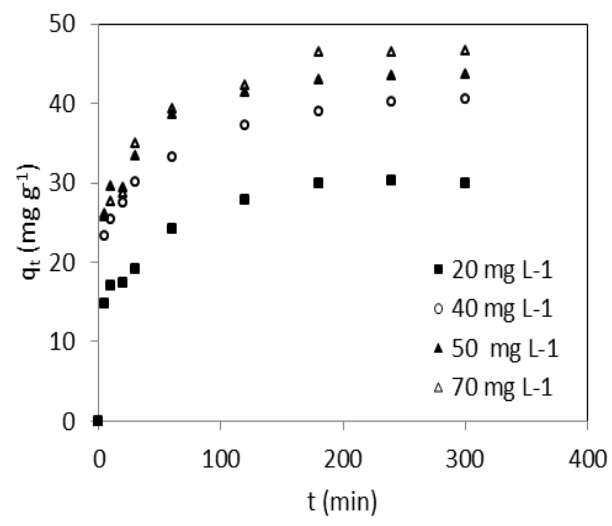

Figura 2 - Efeito do tempo e da concentração inicial de corante sobre a adsorção do corante tartrazina sobre sementes de mamão, a pH 2,5 e $25{ }^{\circ} \mathrm{C}$. 
O perfil da curva do tempo em função da quantidade adsorvida foi uma curva única, suave e contínua levando ao equilíbrio de adsorção. A Figura 2 mostra também que o tempo de contato necessário para atingir o equilíbrio foi de cerca de $100 \mathrm{~min}$ para todas as concentrações iniciais avaliadas.

\subsection{Estudos de equilíbrio}

A análise do processo de adsorção requer um estudo de equilíbrio de adsorção. O teste de equilíbrio de adsorção fornece os dados físico-químicos sobre a aplicabilidade da adsorção. Os dados foram ajustados utilizando as isotermas de Langmuir (Khaled et al., 2009), Freundlich (Khaled et al., 2009) e Temkin (Foo \& Hameed, 2010) (Tabela 1).

Tabela 1 - Modelos de isotermas.

\begin{tabular}{|c|c|c|}
\hline Lanomuir & $\frac{1}{=}=\frac{1}{1}$ & $\begin{array}{l}q_{e} \text { - capacidade de adsorção no equilíbrio }\left(\mathrm{mg} \mathrm{g}^{-1}\right) \\
C_{e} \text { - concentração no equilíbrio }\left(\mathrm{mg} \mathrm{L}^{-1}\right)\end{array}$ \\
\hline Lang & $q_{e} \quad k_{L} q_{\max } C_{e} \quad q_{\max }$ & $\begin{array}{l}q_{\max } \text { - capacidade máxima de adsorção }\left(\mathrm{mg} \mathrm{g}^{-1}\right) \\
k_{L}-\text { constante de equilíbrio de Langmuir }\left(\mathrm{L} \mathrm{mg}^{-1}\right)\end{array}$ \\
\hline Freundlich & )$+\frac{1}{n_{p}} \log \left(C_{\theta}\right)$ & $\begin{array}{l}k_{F} \text { - constante de adsorção de Freundlich }\left(\mathrm{mg} \mathrm{g}^{-1}\right) \\
n_{F} \text { - constante de Freundlich relacionada com a } \\
\text { intensidade da adsorção }\end{array}$ \\
\hline Temkin & $q_{e}=\frac{R T}{B} \ln (A)+\left(\frac{R T}{B}\right) \ln \left(C_{e}\right)$ & $\begin{array}{l}R \text { - constante dos gases ideais }\left(8.314 \mathrm{~J} \mathrm{~mol}^{-1} \mathrm{~K}^{-1}\right) \\
T \text { - temperatura absoluta }(\mathrm{K}) \\
A \text { - constante da ligação no equilíbrio }\left(\mathrm{L} \mathrm{mg}^{-1}\right) \\
B \text { - constante relacionada com o calor de adsorção }\end{array}$ \\
\hline
\end{tabular}

Os parâmetros de adsorção obtidos a partir dos gráficos são mostrados na Figura 3. A isoterma de Langmuir apresentou o melhor ajuste dos dados experimentais, com um coeficiente de determinação de 0,99 , enquanto que os outros modelos mostraram coeficientes de correlação menores. Estes resultados sugerem que houve uma cobertura de monocamada de moléculas do corante sobre a superfície das sementes de mamão. Para o modelo de Langmuir, foi encontrada uma capacidade de adsorção máxima de $51 \mathrm{mg} \mathrm{g}^{-1}$. 
(a)

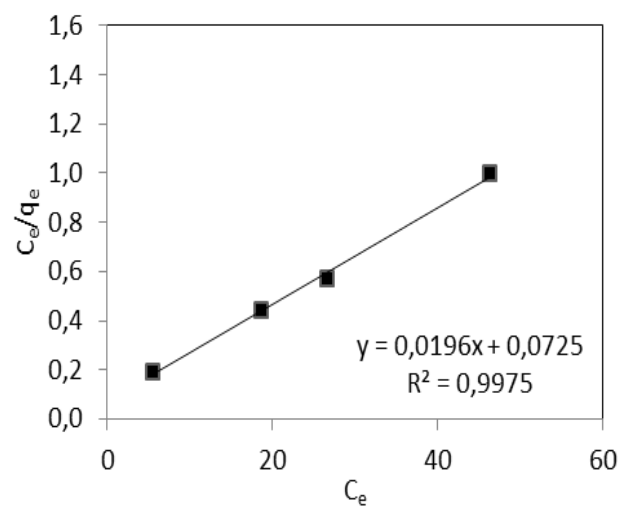

(b)

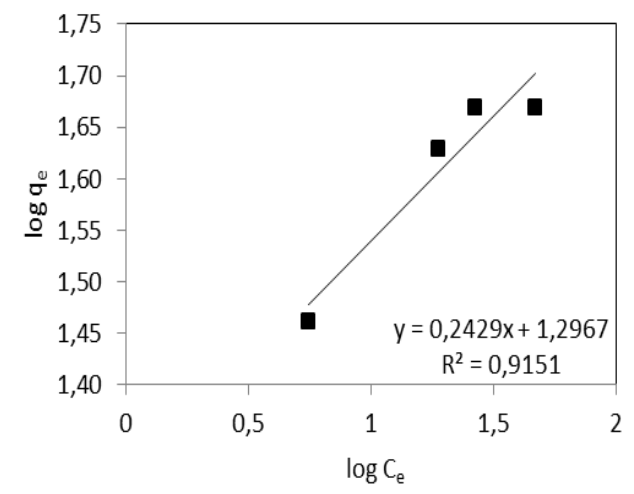

(c)

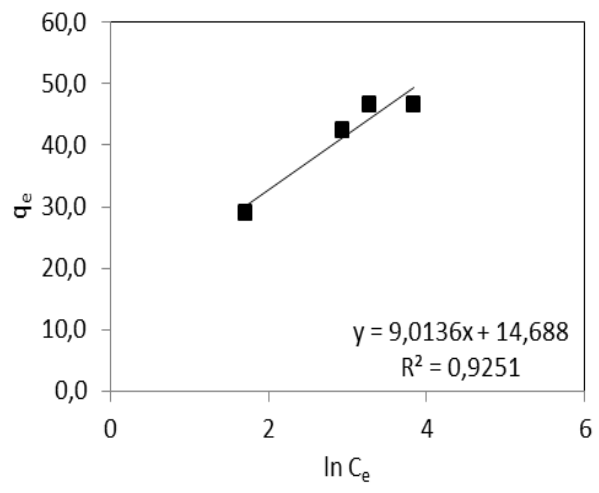

Figura 3 - Isotermas de (a) Langmuir; (b) Freundlich e (c) Temkin para a adsorção do corante tartrazina sobre sementes de mamão.

\subsection{Estudos cinéticos}

O estudo cinético é importante porque fornece informações importantes sobre o mecanismo de adsorção de adsorbatos em solução. Três modelos cinéticos foram testados para ajustar os dados apresentados na Figura 2: pseudo-primeira ordem (Malik, 2004), pseudo-segunda ordem (Malik, 2004) e Elovich (Khaled et al., 2009) (Tabela 2).

Os três modelos cinéticos foram ajustados (mostrados na Figura 4) e os parâmetros cinéticos estão apresentados na Tabela 3. Utilizando o coeficiente de determinação $\left(\mathrm{R}^{2}\right)$ para comparar os modelos, observou-se que a adsorção de corante sobre semente de mamão seguiu o modelo de pseudo-segunda ordem. 
Tabela 2 - Modelos cinéticos de adsorção.

\begin{tabular}{|c|c|c|}
\hline $\begin{array}{c}\text { Pseudo-primeira } \\
\text { ordem }\end{array}$ & $\ln \left(q_{e}-q_{t}\right)=\ln \left(q_{e}\right)-k_{1} t$ & $\begin{array}{l}q_{e} \text { - capacidade no equilíbrio de adsorção }\left(\mathrm{mg} \mathrm{g}^{-1}\right) \\
q_{t}-\text { capacidade de adsorção no tempo } t\left(\mathrm{mg} \mathrm{g}^{-1}\right) \\
k_{l}-\text { constante da taxa de adsorção de pseudo-primeira ordem } \\
\left(\min ^{-1}\right) \\
\mathrm{t}-\text { tempo }(\min )\end{array}$ \\
\hline $\begin{array}{l}\text { Pseudo-segunda } \\
\text { ordem }\end{array}$ & $\frac{1}{q_{t}}=\frac{1}{k_{2} q_{e}^{2}}+\frac{1}{q_{e}} t$ & $\begin{array}{l}k_{2}-\text { constante da taxa de adsorção de pseudo-segunda ordem } \\
\left(\mathrm{g} \mathrm{mg}^{-1} \mathrm{~min}^{-1}\right) \\
h \text { - taxa de adsorção inicial }\left(\mathrm{mg} \mathrm{g}^{-1} \min ^{-1}\right)\end{array}$ \\
\hline Elovich & $\begin{array}{c}h=k_{2} q_{e}^{2} \\
q_{t}=\frac{1}{\beta} \ln (\alpha \beta)+\frac{1}{\beta} \ln (t)\end{array}$ & $\begin{array}{l}\alpha \text { - taxa de adsorção inicial }\left(\mathrm{mg} \mathrm{g}^{-1} \min ^{-1}\right) \\
\beta \text { - constante de dessorção }\left(\mathrm{g} \mathrm{mg}^{-1}\right)\end{array}$ \\
\hline
\end{tabular}
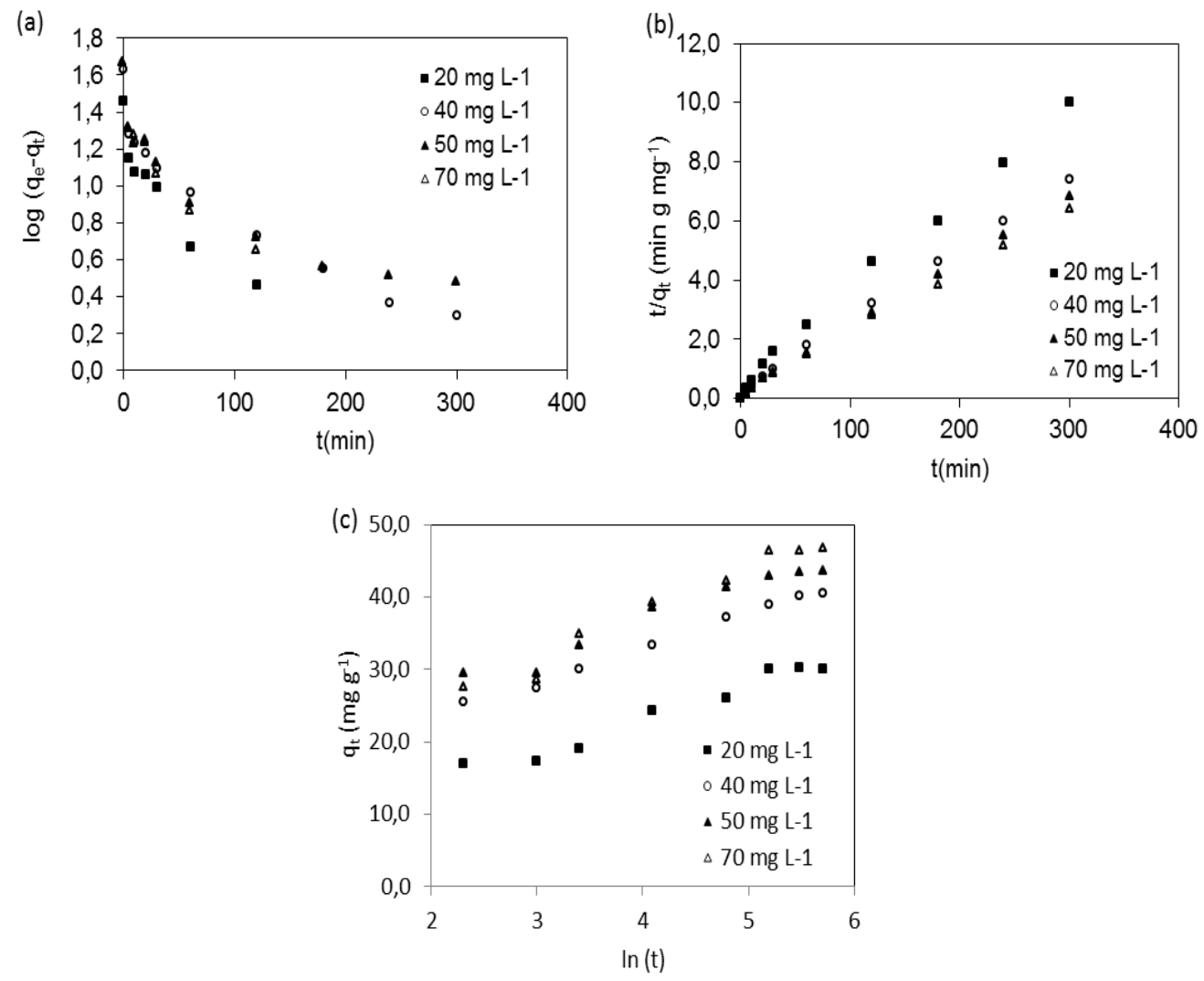

Figura 4 - Modelos de (a) pseudo-primeira ordem; (b) Pseudo-segunda ordem; e (c) Elovich para a adsorção do corante tartrazina sobre sementes de mamão. 
Tabela 3 - Parâmetros cinéticos para a adsorção de corante sobre sementes de mamão, em concentrações de corante de 20 e $70 \mathrm{mg} \mathrm{L}^{-1}$.

\begin{tabular}{|c|c|c|c|}
\hline \multirow{2}{*}{ Modelos } & \multirow{2}{*}{ Parâmetros } & \multicolumn{2}{|c|}{ Tartrazina } \\
\hline & & 20 & 70 \\
\hline \multirow{3}{*}{ Primeira ordem } & $\mathrm{k}_{1}\left(\min ^{-1}\right)$ & 0,024 & 0,016 \\
\hline & $\mathrm{q}_{\mathrm{e}}$ (calc.) & 27,71 & 41,15 \\
\hline & $\mathrm{R}^{2}$ & 0,956 & 0,828 \\
\hline \multirow{3}{*}{ Segunda ordem } & $\mathrm{k}_{2}\left(\mathrm{~g} \mathrm{mg}^{-1} \mathrm{~min}^{-1}\right)$ & 0,003 & 0,002 \\
\hline & $\mathrm{q}_{\mathrm{e}}$ (calc.) & 27,85 & 45,40 \\
\hline & $\mathrm{R}^{2}$ & 0,997 & 0,998 \\
\hline \multirow{4}{*}{ Elovich } & $\beta$ & 0,160 & 0,134 \\
\hline & $\alpha$ & 20,66 & 20,01 \\
\hline & $\mathrm{q}_{\mathrm{e}}$ (calc.) & 31,80 & 49,82 \\
\hline & $\mathrm{R}^{2}$ & 0,957 & 0,929 \\
\hline
\end{tabular}

\section{CONCLUSÕES}

Neste estudo, sementes de mamão foram aplicadas com sucesso para remoção do corante tartrazina a partir da solução aquosa. O estudo de equilíbrio confirmou que a isoterma de Langmuir foi o modelo que melhor ajustou o processo de adsorção. Os estudos cinéticos demonstram que a cinética de pseudo-segunda ordem foi o melhor modelo aplicável. O potencial de adsorção máxima de sementes de mamão como adsorvente para remoção de corante tartrazina foi de $51 \mathrm{mg} \mathrm{g}^{-1}$. Portanto, sementes de mamão possuem potencial para serem aplicadas como adsorvente para remoção do corante tartrazina em soluções aquosas.

\section{REFERÊNCIAS}

FOLETTO, E. L.; WEBER, C. T.; PAZ, D. S.; MAZUTTI, M.A.; MEILI, L.; BASSACO, M. M.; COLLAZZO, G. C. Adsorption of leather dye onto activated carbon prepared from bottle gourd: equilibrium, kinetic and mechanism studies. Water Sci. Technol., v. 67, p. 201-209, 2012.

FOLETTO, E. L.; BATTISTON, S.; MAZUTTI, M. A.; JAHN, S. L. Adsorption of a leather dye on mesoporous struvite obtained from swine wastewater. Chem. Eng. Commun., v. 200, p. 1027-1038, 2013a.

FOLETTO, E. L.; WEBER, C. T.; BERTUOL, D. A.; MAZUTTI, M. A. Application of Papaya Seeds as a Macro-/Mesoporous Biosorbent for the Removal of Large Pollutant Molecule from Aqueous Solution: Equilibrium, Kinetic, and Mechanism Studies. Separ Sc Technol, v. 48, p. 2817-2824, $2013 b$.

FOO, K. Y.; HAMEED, B. H. Insights into the modeling of adsorption isotherm systems. Chem. Eng. J., v. 156 , p. 2-10. 2010.

GUTPA, V. K.; RAJEEV, J.; SHRIVASTAVA, M.; NAYAK, A. Equilibrium and Thermodynamic Studies on the Adsorption of the Dye Tartrazine onto Waste "Coconut Husks" Carbon and Activated Carbon . J. Chem. Eng. Data, v. 55, p. 5083-5090, 2010. 
HAMEED, B. H. Evaluation of papaya seeds as a novel non-conventional low-cost adsorbent for removal of methylene blue. J. Hazard. Mater., v. 162, p. 939-944, 2009.

IOANNOU, Z.; SIMITZIS, J. Adsorption of methylene blue dye onto activated carbons based on agricultural by-products: equilibrium and kinetic studies. Water Sci. Technol., v. 67, p. 1688-1694, 2013.

KHALED, A.; NEMR, A. E.; SIKAILY, A. E.; ABDELWAHAB, O. Removal of Direct N Blue-106 from artificial textile dye effluent using activated carbon from orange peel: adsorption isotherm and kinetic studies. J. Hazar. Mater. , v. 165, 100-110, 2009.

LINHARES, B.; WEBER, C. T.; FOLETTO, E. L.; PAZ, D. S.; MAZUTTI, M. A.; COLLAZZO, G. C. In Press. Activated carbon prepared from yerba mate used as a novel adsorbent for removal of tannery dye from aqueous solution. Environ Technol, v. 34, p. 2401-2406, 2013.

MALIK, P. K. Dye removal from wastewater using activated carbon developed from sawdust: adsorption equilibrium and kinetics. J. Hazar. Mater., v. 113, p. 81-88. 2004.

PAZ, D. S.; BAIOTTO, A.; SCHWAAB, M.; MAZUTTI, M. A.; BASSACO, M. M.; BERTUOL, D.; FOLETTO, E. L.; MEILI, L. Use of papaya seeds as a biosorbent of methylene blue from aqueous solution. Water Sci Technol., v. 68, p. 441-447, 2013.

RAMAKRISHNA, K. R.; VIRARAGHAVAN, T. Dye removal using low cost adsorbents. Water Sci Technol., v. 6, p. 189-196, 1997.

WEBER, C. T.; FOLETTO, E. L; MEILI, L. Removal of Tannery Dye from Aqueous Solution Using Papaya Seed as an Efficient Natural Biosorbent. Water Air Soil Poll., v. 224, p. 1427-1438, 2013.

WENG, C. H.; CHANG, E. E.; CHIANG, P. C. Characteristics of new coccine dye adsorption onto digested sludge particulates. Water Sci Technol., v. 44, p. 279-284, 2001.

YANG, F.; SONG, X.; YAN, L. Preparation of cationic waste paper and its application in poisonous dye removal. Water Sci Technol., v. 67, p. 2560-2567, 2013. 